\title{
The ARL of modified Shewhart control charts for conditionally heteroskedastic models
}

\author{
Esmeralda Gonçalves • Joana Leite • \\ Nazaré Mendes-Lopes
}

Received: date / Accepted: date

\begin{abstract}
In this paper we consider the modified Shewhart control chart for $\mathrm{ARCH}$ processes and introduce it for threshold ARCH (TARCH) ones. For both charts, we determine bounds for the distribution of the in-control run length $(R L)$ and, consequently, for its average $(A R L)$, both depending only on the distribution of the generating white noise, the model parameters and the critical value. For the ARCH model, we compare our bounds with others available in literature and show how they improve the existing ones. We present a simulation study to assess the quality of the bounds calculated for the $A R L$.
\end{abstract}

Keywords Shewhart control chart · average run length · time series · stationarity $\cdot \mathrm{ARCH}$ model $\cdot \mathrm{TARCH}$ model

Mathematics Subject Classification (2000) 62L10 - 62M10 - 60G10

\section{Introduction}

Control charts are an important tool of Statistical Process Control (SPC) extensively used in industry. They allow monitoring whether an observed process diverts from a supposed target process by issuing out-of-control alerts. These schemes were introduced by Shewhart in the 1920's (Shewhart 1931) and his original design is still widely used, despite multiple alternatives that

Esmeralda Gonçalves and Nazaré Mendes-Lopes

Department of Mathematics of the University of Coimbra - FCTUC

Apartado 3008, 3001-454 Coimbra, Portugal

Joana Leite

Institute of Accounting and Administration of Coimbra - IPC

Quinta Agrícola - Bencanta, 3040-316 Coimbra, Portugal

Tel.: +351-239802000

Fax: +351-239445445

E-mail: jleite@iscac.pt 
have appeared in literature, like EWMA and CUSUM charts. Several studies show that Shewhart type charts are still the best to detect large shifts.

In the last decades, the applicability of control schemes has been extended from independent processes to time series, namely, with the appearance of charts which incorporate the time series structure into its design, named modified charts. Vasilopoulos and Stamboulis (1978) were the precursors, suggesting the modified Shewhart chart for AR processes. The first modified chart for conditionally heteroskedastic models was presented by Severin and Schmid (1999), who considered the generalized ARCH model. The conditionally heteroskedastic models are particulary well-suited for modeling financial time series, so combining them with control charts opens the door to the use of SPC techniques also to practitioners of the financial field.

Given the variety of control charts available to monitor a time series, raises the question of evaluating the best design to detect a deviation from target as soon as possible. The average run length $(A R L)$ is widely used as a performance measure for control charts and, when dealing with time series, is defined as the average number of instants that must go by before one indicates an out-of-control condition.

Formally, let $Y=\left(Y_{t}, t \in \mathbb{Z}\right.$ ) be the target process, (weakly) stationary, such that $E\left(Y_{t}\right)=\mu_{0}$ and $V\left(Y_{t}\right)=\sigma_{Y}^{2}$. The observed process $X=\left(X_{t}, t \in \mathbb{N}\right)$ is in-control if $X_{t}=Y_{t}$, for $t \geq 1$. For the modified Shewhart chart, given the critical value $c \in \mathbb{R}^{+}, X$ is said to be out-of-control at time $t$ if $\left|X_{t}-\mu_{0}\right|>c \sigma_{Y}$ (Schmid 1995). Consequently, we have $A R L=E\left(N_{S}\right)$, where $N_{S}$ is the run length $(R L)$ of the modified Shewhart chart defined as

$$
N_{S}=\inf \left\{t \in \mathbb{N}:\left|X_{t}-\mu_{0}\right|>c \sigma_{Y}\right\} .
$$

The $A R L$ is also important to set the design of the control chart, since $c$ is often taken so that the in-control $A R L$ is equal to a specified constant. For example, Severin and Schmid (1999) state that, in financial applications using daily stock market values, the in-control $A R L$ should be 20,40 or 60 , because these values correspond to 1,2 or 3 months of stock market, respectively, in this way conditioning the value of $c$.

The primary goal of this paper is to improve or derive theoretical bounds for the in-control $A R L$ of the modified Shewhart chart for two classes of conditionally heteroskedastic models: the ARCH model (Engle 1982) and the threshold ARCH (TARCH) model (Zakoian 1994). Whenever possible we will compare the $A R L$ of these charts with the $A R L$ of classical Shewhart charts, which allows to realize what happens if the process is wrongly assumed independent.

The remainder of this article is organized as follows. Section 2 introduces the two conditionally heteroskedastic models which are taken into account in this study. Section 3 is dedicated to the ARCH model. We start this section with an overview of existing bounds for $P\left(N_{S}>n\right)$, obtained by Severin and Schmid (1999) and Pawlak and Schmid (2001), and derive a new lower bound for this probability following the method present in Gonçalves and MendesLopes (2007). This enables us to calculate the corresponding bounds for the 
$A R L$, which we then compare. In section 4, we introduce the modified Shewhart chart for TARCH processes and determine bounds for $P\left(N_{S}>n\right)$ and, consequently, for $A R L$. Section 5 provides a simulation study to assess the quality of the bounds calculated for the $A R L$ by comparing them with the estimated $A R L$. In this paper, we will make use of the following conventions: $\sum_{i=1}^{0}(\cdot)=0$ and $\prod_{t=2}^{1}(\cdot)=1$.

\section{The $\operatorname{ARCH}(q)$ and $\operatorname{TARCH}(q)$ models}

In conditionally heteroskedastic models, the real stochastic process $Y=\left(Y_{t}, t \in \mathbb{Z}\right)$ is set to be, for every $t \in \mathbb{Z}$,

$$
Y_{t}=Z_{t} \sigma_{t}
$$

where $Z=\left(Z_{t}, t \in \mathbb{Z}\right)$ is a sequence of independent and identically distributed (iid) real random variables, with zero mean and unit variance, such that $Z_{t}$ is independent of the $\sigma$-field generated by the past of $Y, \underline{Y}_{t-1}=\sigma\left(Y_{t-1}, Y_{t-2}, \ldots\right)$, and where $\sigma_{t}^{2}=V\left(Y_{t} \mid \underline{Y}_{t-1}\right)$ is a measurable function of past observations of $Y$.

The specification of $\sigma_{t}$ determines the model. When $Y$ is an $\operatorname{ARCH}(q)$ process, we set

$$
\sigma_{t}^{2}=\alpha_{0}+\sum_{i=1}^{q} \alpha_{i} Y_{t-i}^{2}
$$

with $\alpha_{0}>0$ and $\alpha_{i} \geq 0(i=1, \ldots, q)$. When $Y$ is a $\operatorname{TARCH}(q)$ process, we specify

$$
\sigma_{t}=\alpha_{0}+\sum_{i=1}^{q} \alpha_{i} Y_{t-i}^{+}-\sum_{i=1}^{q} \beta_{i} Y_{t-i}^{-}
$$

where $Y_{t}^{+}=Y_{t} \mathbb{1}_{\left\{Y_{t} \geq 0\right\}}, Y_{t}^{-}=Y_{t} \mathbb{1}_{\left\{Y_{t}<0\right\}}$ and with $\alpha_{0}>0, \alpha_{i} \geq 0, \beta_{i} \geq 0$ $(i=1, \ldots, q)$.

Regarding the specification of $\sigma_{t}$, the main difference between these two models is the ability which only the TARCH model has to take into account different reactions of volatility according to the sign of past values of the process. Nevertheless, both models are well-established among practitioners particularly of the financial field.

Our aim to evaluate the in-control $A R L$ requires that we work with stationary processes. We observe that, under weak stationarity conditions, the conditionally heteroskedastic models are centered and non correlated processes. A necessary and sufficient condition of stationarity for the $\operatorname{ARCH}(q)$ model is $\sum_{i=1}^{q} \alpha_{i}<1$. Under this condition, the variance of $Y, V\left(Y_{t}\right)=\sigma_{Y}^{2}$, exists and is equal to

$$
\sigma_{Y}^{2}=\frac{\alpha_{0}}{1-\sum_{i=1}^{q} \alpha_{i}}
$$


For the generalized TARCH (GTARCH) model, the necessary and sufficient condition of stationarity depends not only on the parameters of the model but also on the moments of $Z_{t}^{+}$and $Z_{t}^{-}$(Gonçalves and Mendes-Lopes 1994). For the $\operatorname{TARCH}(1)$ process, the condition is $E\left[\left(\alpha_{1} Z_{t}^{+}-\beta_{1} Z_{t}^{-}\right)^{2}\right]<1$ and the variance of $Y, V\left(Y_{t}\right)=\sigma_{Y}^{2}$, exists and is equal to

$$
\sigma_{Y}^{2}=\frac{\alpha_{0}^{2}\left[1+E\left(\alpha_{1} Z_{t}^{+}-\beta_{1} Z_{t}^{-}\right)\right]}{\left[1-E\left(\alpha_{1} Z_{t}^{+}-\beta_{1} Z_{t}^{-}\right)\right]\left\{1-E\left[\left(\alpha_{1} Z_{t}^{+}-\beta_{1} Z_{t}^{-}\right)^{2}\right]\right\}} .
$$

Remark 1 In both formulations of $\sigma_{t}$, when all parameters, apart from $\alpha_{0}$, are equal to zero, the model is no longer conditionally heteroskedastic. In this case $Y$ is an iid process and $N_{S}$ follows the geometric law $G(\theta)$, with support $\mathbb{N}$ and $\theta=1-F_{|Z|}(c)=1-F_{Z^{2}}\left(c^{2}\right)$, where $F_{|Z|}$ and $F_{Z^{2}}$ are the distribution functions of $\left|Z_{t}\right|$ and $Z_{t}^{2}$, respectively. Thus, in the in-control state,

$$
P_{i i d}\left(N_{S}>n\right)=(1-\theta)^{n}, n=0,1,2, \ldots, \quad \text { and } \quad A R L_{i i d}=\frac{1}{\theta}
$$

with the index "iid" meaning that the target process $Y$ is an iid process.

\section{In-control $A R L$ of modified Shewhart chart for the ARCH process}

In this section we assume that the target process $Y$ follows a stationary $\operatorname{ARCH}(q)$ model and that the observed process $X$ is in-control. So,

$$
P\left(N_{S}>n\right)=P\left(\left|Y_{1}\right| \leq c \sigma_{Y}, \ldots,\left|Y_{n}\right| \leq c \sigma_{Y}\right), n=1,2, \ldots .
$$

We note that $P\left(N_{S}>0\right)=1$.

3.1 Bounds for $P_{n}=P\left(N_{S}>n\right)$

The first bounds for the $P_{n}=P\left(N_{S}>n\right)$ were derived by Severin and Schmid (1999). Their upper bound,

$$
\underset{\operatorname{ARCH}(q)}{U B_{1} P_{n}}=\left[F_{Z^{2}}\left(\frac{c^{2} \sigma_{Y}^{2}}{\alpha_{0}}\right)\right]^{n},
$$

valid for every $c \in \mathbb{R}^{+}$and obtained by an elementary technique, is simple to use but its performance is not always good. Nevertheless, it is the unique known in the literature until now. In what concerns their lower bound,

$$
\underset{\operatorname{ARCH}(q)}{L B_{1} P_{n}}=P\left(N_{S}>\min \{q, n\}\right) \times\left[F_{Z^{2}}\left(\frac{c^{2}}{1+\left(c^{2}-1\right) \sum_{i=1}^{q} \alpha_{i}}\right)\right]^{\max \{0, n-q\}}
$$


also valid for every $c \in \mathbb{R}^{+}$, we remark that it depends on the probability in evaluation. This problem was firstly addressed by Pawlak and Schmid (2001), who obtained the following lower bound depending only on the distribution function of $Z_{t}^{2}$ and the parameters of the model, considering $Z_{t}$ absolutely continuous with a differentiable density of probability $f_{Z}$ :

$$
\underset{\operatorname{ARCH}(q)}{L B_{2} P_{n}}=\left[\prod_{t=1}^{\min \{q, n\}} F_{Z^{2}}\left(\frac{c^{2}}{1+\left(c^{2}-1\right) \sum_{i=1}^{t-1} \alpha_{i}}\right)\right] \times\left[F_{Z^{2}}\left(c^{2}\right)\right]^{\max \{0, n-q\}},
$$

valid for every $c \in \mathbb{R}^{+}$such that $2 f_{Z^{2}}(x)+x f_{Z^{2}}^{\prime}(x) \geq 0$, where $x=\frac{c^{2} \sigma_{Y}^{2}}{\alpha_{0}+u}$, with $u \geq 0$.

We also consider this matter following the method proposed in Gonçalves and Mendes-Lopes (2007) for generalized TARCH processes. We begin by deriving a lower bound for the laws of finite dimension for the process $Y^{2}$. The proof of this result is set in the appendix.

Theorem 1 Let $Y=\left(Y_{t}, t \in \mathbb{Z}\right)$ be an $A R C H(q)$ process such that $Z_{t}$ is absolutely continuous with a differentiable density of probability $f_{Z}$. Then, if $Y$ is stationary, it holds:

(a) for $n \leq q$,

$P\left(Y_{t}^{2} \leq x_{t}, t=1, \ldots, n\right) \geq F_{Z^{2}}\left(\frac{x_{1}}{\sigma_{Y}^{2}}\right) \prod_{t=2}^{n} F_{Z^{2}}\left(\frac{x_{t}}{\alpha_{0}+\sum_{i=1}^{t-1} \alpha_{i} x_{t-i}+\sigma_{Y}^{2} \sum_{i=t}^{q} \alpha_{i}}\right)$

(b) for $n>q$,

$P\left(Y_{t}^{2} \leq x_{t}, t=1, \ldots, n\right) \geq F_{Z^{2}}\left(\frac{x_{1}}{\sigma_{Y}^{2}}\right) \times$

$$
\times \prod_{t=2}^{q} F_{Z^{2}}\left(\frac{x_{t}}{\alpha_{0}+\sum_{i=1}^{t-1} \alpha_{i} x_{t-i}+\sigma_{Y}^{2} \sum_{i=t}^{q} \alpha_{i}}\right) \times \prod_{t=q+1}^{n} F_{Z^{2}}\left(\frac{x_{t}}{\alpha_{0}+\sum_{i=1}^{q} \alpha_{i} x_{t-i}}\right)
$$

in both cases, for all $\left.\left(x_{1}, \ldots, x_{n}\right) \in\right] 0,+\infty\left[^{n}\right.$ such that

$$
2 f_{Z^{2}}\left(\frac{x_{t}}{\alpha_{0}+u}\right)+\frac{x_{t}}{\alpha_{0}+u} f_{Z^{2}}^{\prime}\left(\frac{x_{t}}{\alpha_{0}+u}\right) \geq 0, t=1, \ldots, \min \{q, n\}
$$

with $u$ any non-negative real number. 
Taking into account that, for the in-control state, we have

$$
P\left(N_{S}>n\right)=P\left(Y_{t}^{2} \leq c^{2} \sigma_{Y}^{2}, t=1, \ldots, n\right),
$$

the previous theorem will be useful considering $x_{1}=\ldots=x_{n}=c^{2} \sigma_{Y}^{2}$. Using the fact that $\sigma_{Y}^{2}=\frac{\alpha_{0}}{1-\sum_{i=1}^{q} \alpha_{i}}$, a new lower bound for $P\left(N_{S}>n\right), n \in \mathbb{N}$, is deduced from theorem 1 .

Corollary 1 Under the conditions of the previous theorem, a lower bound for $P_{n}$ is

$$
\begin{aligned}
\underset{\operatorname{ARCH}(q)}{L B_{3} P_{n}=} & \prod_{t=1}^{\min \{q, n\}} F_{Z^{2}}\left(\frac{c^{2}}{1+\left(c^{2}-1\right) \sum_{i=1}^{t-1} \alpha_{i}}\right) \times \\
& \times\left[F_{Z^{2}}\left(\frac{c^{2}}{1+\left(c^{2}-1\right) \sum_{i=1}^{q} \alpha_{i}}\right)\right]^{\max \{0, n-q\}}
\end{aligned}
$$

which is valid for every $c \in \mathbb{R}^{+}$such that $2 f_{Z^{2}}(x)+x f_{Z^{2}}^{\prime}(x) \geq 0$, where $x=\frac{c^{2} \sigma_{Y}^{2}}{\alpha_{0}+u}$, with $u \geq 0$.

We note that, when $\alpha_{1}=\ldots=\alpha_{q}=0$, we have

$$
\underset{\operatorname{ARCH}(q)}{U B_{1} P_{n}}=\underset{\operatorname{ARCH}(q)}{L B_{1} P_{n}}=\underset{\operatorname{ARCH}(q)}{L B_{2} P_{n}}=\underset{\operatorname{ARCH}(q)}{L B_{3} P_{n}}=\left[F_{Z^{2}}\left(c^{2}\right)\right]^{n}=P_{i i d}\left(N_{S}>n\right)
$$

3.2 Bounds for the $A R L$

The bounds for the $A R L$ are determined using the bounds for $P\left(N_{S}>n\right)$, considering that

$$
A R L=E\left(N_{S}\right)=\sum_{n=1}^{+\infty} n P\left(N_{S}=n\right)=\sum_{n=0}^{+\infty} P\left(N_{S}>n\right) .
$$

Starting from $\underset{\operatorname{ARCH}(q)}{U B_{1} P_{n}}$ and $\underset{\operatorname{ARCH}(q)}{L B_{1} P_{n}}$, Severin and Schmid (1999) deduced, respectively, the following upper bound for the in-control $A R L$

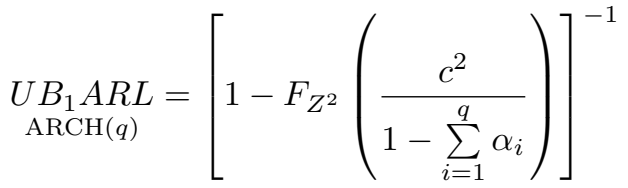


and the lower bound for the in-control $A R L$

$$
\underset{\operatorname{ARCH}(q)}{L B_{1} A R L}=1+\sum_{n=1}^{q-1} P\left(N_{S}>n\right)+\frac{P\left(N_{S}>q\right)}{1-F_{Z^{2}}\left(\frac{c^{2}}{1+\left(c^{2}-1\right) \sum_{i=1}^{q} \alpha_{i}}\right)}
$$

with both bounds valid for every $c \in \mathbb{R}^{+}$.

Using the two other lower bounds for $P\left(N_{S}>n\right), \underset{\operatorname{ARCH}(q)}{L B_{2} P_{n}}$ and $\underset{\operatorname{ARCH}(q)}{L B_{3} P_{n}}$, we have the following result:

Corollary 2 Under the conditions of theorem 1,

$$
\begin{aligned}
\underset{\mathrm{ARCH}(q)}{L B_{2} A R L}=1 & +\sum_{n=1}^{q-1} \prod_{t=1}^{n} F_{Z^{2}}\left(\frac{c^{2}}{1+\left(c^{2}-1\right) \sum_{i=1}^{t-1} \alpha_{i}}\right)+ \\
+ & {\left[\prod_{t=1}^{q} F_{Z^{2}}\left(\frac{c^{2}}{1+\left(c^{2}-1\right) \sum_{i=1}^{t-1} \alpha_{i}}\right)\right] \frac{1}{1-F_{Z^{2}}\left(c^{2}\right)} }
\end{aligned}
$$

and

$$
\begin{aligned}
\underset{\operatorname{ARCH}(q)}{L B_{3} A R L} & =1+\sum_{n=1}^{q-1} \prod_{t=1}^{n} F_{Z^{2}}\left(\frac{c^{2}}{1+\left(c^{2}-1\right) \sum_{i=1}^{t-1} \alpha_{i}}\right)+ \\
& +\left[\prod_{t=1}^{q} F_{Z^{2}}\left(\frac{c^{2}}{1+\left(c^{2}-1\right) \sum_{i=1}^{t-1} \alpha_{i}}\right)\right] \frac{1}{1-F_{Z^{2}}\left(\frac{c^{2}}{1+\left(c^{2}-1\right) \sum_{i=1}^{q} \alpha_{i}}\right)}
\end{aligned}
$$

are lower bounds for the $A R L$, for every $c \in \mathbb{R}^{+}$such that $2 f_{Z^{2}}(x)+x f_{Z^{2}}^{\prime}(x) \geq$ 0 , where $x=\frac{c^{2} \sigma_{Y}^{2}}{\alpha_{0}+u}$, with $u \geq 0$.

In the following, we analyse in some particular cases the regions where these bounds are valid.

Example 1 If $Z_{t}$ follows the standard normal distribution, then, for $x>0$,

$$
f_{Z^{2}}(x)=\frac{1}{\sqrt{2 \pi}} \times \frac{e^{-x / 2}}{x^{1 / 2}}
$$


and

$$
2 f_{Z^{2}}(x)+x f_{Z^{2}}^{\prime}(x)=f_{Z^{2}}(x)\left[2+x\left(-\frac{1}{2}-\frac{1}{2 x}\right)\right] .
$$

Consequently

$$
2 f_{Z^{2}}(x)+x f_{Z^{2}}^{\prime}(x) \geq 0 \Leftrightarrow x \leq 3 .
$$

As $x=\frac{c^{2} \sigma_{Y}^{2}}{\alpha_{0}+u}$, with $u \geq 0$, it holds that $x \leq 3$ if $\frac{c^{2} \sigma_{Y}^{2}}{\alpha_{0}} \leq 3$. Then for

$$
0<c \leq \frac{\sqrt{3 \alpha_{0}}}{\sigma_{Y}}=\sqrt{3\left(1-\sum_{i=1}^{q} \alpha_{i}\right)}
$$

$L B_{2} A R L$ and $L B_{3} A R L$ are lower bounds for the in-control $A R L$. $\operatorname{ARCH}(q) \quad \operatorname{ARCH}(q)$

Example 2 If $Z_{t}$ follows the unit variance distribution based on the Student's t-distribution with $\alpha>2$ degrees of freedom, then

$$
f_{Z}(x)=\frac{1}{\sqrt{(\alpha-2) \pi}} \cdot \frac{\Gamma\left(\frac{\alpha+1}{2}\right)}{\Gamma\left(\frac{\alpha}{2}\right)} \cdot\left(1+\frac{x^{2}}{\alpha-2}\right)^{-\frac{\alpha+1}{2}} .
$$

So, for $x>0, f_{Z^{2}}(x)=\frac{1}{2 \sqrt{x}}\left[f_{Z}(\sqrt{x})+f_{Z}(-\sqrt{x})\right]=\frac{1}{\sqrt{(\alpha-2) \pi x}} \cdot \frac{\Gamma\left(\frac{\alpha+1}{2}\right)}{\Gamma\left(\frac{\alpha}{2}\right)}$. $\left(1+\frac{x}{\alpha-2}\right)^{-\frac{\alpha+1}{2}}$. Hence

$$
2 f_{Z^{2}}(x)+x f_{Z^{2}}^{\prime}(x)=f_{Z^{2}}(x)\left[2-\frac{\alpha-2+2 x+\alpha x}{2(\alpha-2+x)}\right] .
$$

As $\alpha>2$, we get

$$
2 f_{Z^{2}}(x)+x f_{Z^{2}}^{\prime}(x) \geq 0 \Leftrightarrow x \leq 3 .
$$

Thus, just like in the previous case, $L B_{2} A R L$ and $L B_{3} A R L$ are lower bounds for the in-control $A R L$, if

$$
0<c \leq \frac{\sqrt{3 \alpha_{0}}}{\sigma_{Y}}=\sqrt{3\left(1-\sum_{i=1}^{q} \alpha_{i}\right)}
$$

3.3 Comparing the lower bounds for the $A R L$

In this section, we show that, for $0<c \leq 1$ such that $2 f_{Z^{2}}(x)+x f_{Z^{2}}^{\prime}(x) \geq 0$, where $x=\frac{c^{2} \sigma_{Y}^{2}}{\alpha_{0}+u}$, with $u \geq 0$, it holds that

$$
\underset{\operatorname{ARCH}(q)}{L B_{1} A R L} \geq \underset{\operatorname{ARCH}(q)}{L B_{3} A R L} \geq \underset{\operatorname{ARCH}(q)}{L B_{2} A R L} \geq A R L_{i i d} .
$$


In fact, as $P\left(N_{S}>n\right) \geq \underset{\operatorname{ARCH}(q)}{L B_{3} P_{n}}$, for all $n \in \mathbb{N}$, then

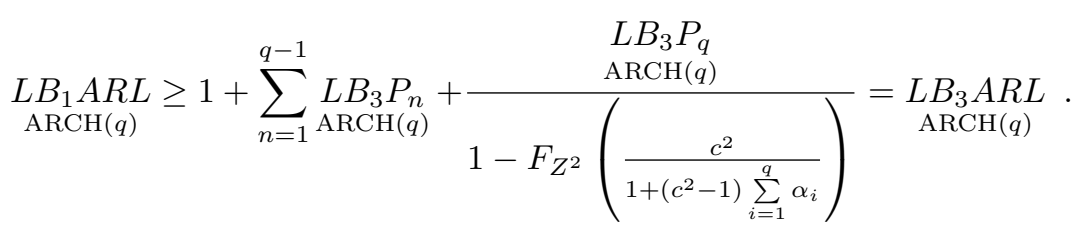

Since, for $0<c \leq 1$ we have $\frac{c^{2}}{1+\left(c^{2}-1\right) \sum_{i=1}^{q} \alpha_{i}} \geq c^{2}$, thus

$$
\frac{1}{1-F_{Z^{2}}\left(\frac{c^{2}}{1+\left(c^{2}-1\right) \sum_{i=1}^{q} \alpha_{i}}\right)} \geq \frac{1}{1-F_{Z^{2}}\left(c^{2}\right)} \Leftrightarrow \underset{\operatorname{ARCH}(q)}{\operatorname{LB}(q)} \underset{\operatorname{ARCH}(q)}{\operatorname{LB}\left(B_{2} A R L\right.}
$$

Finally, for $0<c \leq 1$, it holds

$$
\underset{\operatorname{ARCH}(q)}{L B_{2} A R L} \geq 1+\sum_{n=1}^{q-1} \prod_{t=1}^{n} F_{Z^{2}}\left(c^{2}\right)+\left[\prod_{t=1}^{q} F_{Z^{2}}\left(c^{2}\right)\right] \frac{1}{1-F_{Z^{2}}\left(c^{2}\right)}=A R L_{i i d} .
$$

As $L B_{1} A R L$ depends on the probability in evaluation, we point out that for $\operatorname{ARCH}(q)$

small critical values $(0<c \leq 1)$, the best lower bound for the in-control $A R L$ is $L B_{3} A R L$. Furthermore, we can state that if the analyst falsely assumes $\operatorname{ARCH}(q)$

the process to be independent, then, for small values of $c$, the $A R L$ for an $\operatorname{ARCH}(q)$ process is always greater than in the iid case.

\section{In-control $A R L$ of modified Shewhart chart for the TARCH process}

In this section we assume that the target process $Y$ follows a stationary $\operatorname{TARCH}(q)$ model and that the observed process $X$ is in-control.

Bounds for the $A R L$ of a TARCH model are obtained considering the work of Gonçalves and Mendes-Lopes (2007).

4.1 Bounds for $P_{n}=P\left(N_{S}>n\right)$

In what concerns the upper bound of $P_{n}$, it is straightforward that

$$
\underset{\operatorname{TARCH}(q)}{U B_{1} P_{n}}=\prod_{t=1}^{n} F_{|Z|}\left(\frac{c \sigma_{Y}}{\alpha_{0}}\right)=\left[F_{|Z|}\left(\frac{c \sigma_{Y}}{\alpha_{0}}\right)\right]^{n}
$$

is an upper bound for $P\left(N_{S}>n\right)$, valid for every $c \in \mathbb{R}^{+}$. 
In order to determine a lower bound for $P\left(N_{S}>n\right)$, we present a new version of theorem 4 of Gonçalves and Mendes-Lopes (2007), in which a smaller and, therefore, better upper bound for $\sigma_{t}$ is considered, introducing $\phi_{i}=$ $\max \left\{\alpha_{i}, \beta_{i}\right\}$, for $i=1, \ldots, q$. The proof of the following result is omitted due to the similarity with the proof of theorem 1.

Theorem 2 Let $Y=\left(Y_{t}, t \in \mathbb{Z}\right)$ be a TARCH $(q)$ process such that $Z_{t}$ is absolutely continuous with a differentiable density probability $f_{Z}$. Then, if $Y$ is stationary, it holds:

(a) for $n \leq q$,

$$
P\left(\left|Y_{t}\right| \leq x_{t}, t=1, \ldots, n\right) \geq F_{|Z|}\left(\frac{x_{1}}{\sigma_{Y}}\right) \prod_{t=2}^{n} F_{|Z|}\left(\frac{x_{t}}{\alpha_{0}+\sum_{i=1}^{t-1} \phi_{i} x_{t-i}+\sigma_{Y} \sum_{i=t}^{q} \phi_{i}}\right),
$$

(b) for $n>q$,

$$
\begin{aligned}
& P\left(\left|Y_{t}\right| \leq x_{t}, t=1, \ldots, n\right) \geq F_{|Z|}\left(\frac{x_{1}}{\sigma_{Y}}\right) \times \\
& \quad \times \prod_{t=2}^{q} F_{|Z|}\left(\frac{x_{t}}{\alpha_{0}+\sum_{i=1}^{t-1} \phi_{i} x_{t-i}+\sigma_{Y} \sum_{i=t}^{q} \phi_{i}}\right) \times \prod_{t=q+1}^{n} F_{|Z|}\left(\frac{x_{t}}{\alpha_{0}+\sum_{i=1}^{q} \phi_{i} x_{t-i}}\right),
\end{aligned}
$$

in both cases, for all $\left.\left(x_{1}, \ldots, x_{n}\right) \in\right] 0,+\infty\left[^{n}\right.$ such that

$$
2 f_{|Z|}\left(\frac{x_{t}}{\alpha_{0}+u}\right)+\frac{x_{t}}{\alpha_{0}+u} f_{|Z|}^{\prime}\left(\frac{x_{t}}{\alpha_{0}+u}\right) \geq 0, t=1, \ldots, \min \{q, n\},
$$

where $u$ is any non-negative real number.

For the in-control state, $P\left(N_{S}>n\right)=P\left(\left|Y_{t}\right| \leq c \sigma_{Y}, t=1, \ldots, n\right)$ and using this theorem with $x_{1}=\ldots=x_{n}=c \sigma_{Y}$, we have the following lower bound for $P_{n}$,

$$
\begin{gathered}
\underset{t=2}{L B_{1} P_{n}=F_{|Z|}(c) \prod_{\operatorname{TARCH}(q)}^{\min \{q, n\}}} F_{|Z|}\left(\frac{c}{\frac{\alpha_{0}}{\sigma_{Y}}+c \sum_{i=1}^{t-1} \phi_{i}+\sum_{i=t}^{q} \phi_{i}}\right) \times \\
\times\left[F_{|Z|}\left(\frac{c}{\frac{\alpha_{0}}{\sigma_{Y}}+c \sum_{i=1}^{q} \phi_{i}}\right)\right]^{\max \{0, n-q\}}
\end{gathered}
$$

valid for every $c \in \mathbb{R}^{+}$such that $2 f_{|Z|}(x)+x f_{|Z|}^{\prime}(x) \geq 0$, where $x=\frac{c \sigma_{Y}}{\alpha_{0}+u}$, with $u \geq 0$.

We observe that, if $\alpha_{1}=\ldots=\alpha_{q}=0$, considering remark 1 , then

$$
\underset{\operatorname{TARCH}(q)}{U B_{1} P_{n}}=\underset{\operatorname{TARCH}(q)}{L B_{1} P_{n}}=\left[F_{|Z|}(c)\right]^{n}=P_{i i d}\left(N_{S}>n\right) .
$$


4.2 Bounds for the $A R L$

Considering $U B_{1} P_{n}$ and $L B_{1} P_{n}$, respectively, we derive the following upper $\operatorname{TARCH}(q) \quad \operatorname{TARCH}(q)$

bound for the in-control $A R L$

$$
\underset{\operatorname{TARCH}(q)}{U B_{1} A R L}=\frac{1}{1-F_{|Z|}\left(\frac{c \sigma_{Y}}{\alpha_{0}}\right)}
$$

valid for every $c \in \mathbb{R}^{+}$, and lower bound for the in-control $A R L$

$$
\begin{array}{r}
\underset{\operatorname{TARCH}(q)}{L B_{1} A R L=}=1+F_{|Z|}(c) \sum_{n=1}^{q-1} \prod_{t=2}^{n} F_{|Z|}\left(\frac{c}{\frac{\alpha_{0}}{\sigma_{Y}}+c \sum_{i=1}^{t-1} \phi_{i}+\sum_{i=t}^{q} \phi_{i}}\right)+ \\
+\frac{F_{|Z|}(c) \prod_{t=2}^{q} F_{|Z|}\left(\frac{c}{\frac{\alpha_{0}}{\sigma_{Y}}+c \sum_{i=1}^{t-1} \phi_{i}+\sum_{i=t}^{q} \phi_{i}}\right)}{1-F_{|Z|}\left(\frac{c}{\frac{\alpha_{0}}{\sigma_{Y}}+c \sum_{i=1}^{q} \phi_{i}}\right)}
\end{array}
$$

valid for every $c \in \mathbb{R}^{+}$such that $2 f_{|Z|}(x)+x f_{|Z|}^{\prime}(x) \geq 0$, where $x=\frac{c \sigma_{Y}}{\alpha_{0}+u}$, with $u \geq 0$. For example, for a TARCH(1) model, we have

$$
\underset{\operatorname{TARCH}(1)}{L B_{1} A R L}=1+\frac{F_{|Z|}(c)}{1-F_{|Z|}\left(\frac{c}{\frac{\alpha_{0}}{\sigma_{Y}}+c \max \left\{\alpha_{1}, \beta_{1}\right\}}\right)} .
$$

We analyse now the regions where these bounds are valid for the same distributions of $Z_{t}$ considered in the $\mathrm{ARCH}$ case. The results are summarized in the next example.

Example 3

(a) Considering that $Z_{t}$ follows the standard normal distribution, then, for $x>0$,

$$
f_{|Z|}(x)=f_{Z}(x)+f_{Z}(-x)=\frac{2 e^{-x^{2} / 2}}{\sqrt{2 \pi}} .
$$

Hence, for $x>0$,

$$
2 f_{|Z|}(x)+x f_{|Z|}^{\prime}(x) \geq 0 \Leftrightarrow 0<x \leq \sqrt{2} .
$$
So, for $0<c \leq \frac{\sqrt{2} \alpha_{0}}{\sigma_{Y}}, \underset{\operatorname{TARCH}(q)}{L B_{1} A R L}$ is a lower bound for the in-control $A R L$. 
(b) Suppose $Z_{t}$ follows the unit variance distribution based on the Student's t-distribution with $\alpha>2$ degrees of freedom as in example 2. So, for $x>0$, $f_{|Z|}(x)=2 f_{Z}(x)$ and $f_{|Z|}^{\prime}(x)=f_{|Z|}(x) \cdot \frac{-(\alpha+1) x}{\alpha-2+x^{2}}$. Hence, for $x>0$,

$$
2 f_{|Z|}(x)+x f_{|Z|}^{\prime}(x) \geq 0 \Leftrightarrow 0<x \leq \sqrt{\frac{2(\alpha-2)}{\alpha-1}} .
$$

Thus $L B_{1} A R L$ is a lower bound for the in-control $A R L$, if $\operatorname{TARCH}(q)$

$$
0<c \leq \sqrt{\frac{2(\alpha-2)}{\alpha-1}} \cdot \frac{\alpha_{0}}{\sigma_{Y}} .
$$

In the next remark we analyse the particular case of the TARCH(1) model and find a region for the critical value for which we can ensure that the $A R L$ is always greater than in the iid case.

Remark 2 Let us consider $q=1$. In this case, the expression of $\sigma_{Y}^{2}$ is given in (6). Then

$$
\sigma_{Y}^{2} \leq \frac{\alpha_{0}^{2}\left(1+\phi_{1}\right)}{\left(1-\phi_{1}\right)\left(1-\phi_{1}^{2}\right)}=\frac{\alpha_{0}^{2}}{\left(1-\phi_{1}\right)^{2}},
$$

since $\alpha_{1} \leq \phi_{1}$ and $\beta_{1} \leq \phi_{1}$, so, for every $t \in \mathbb{Z}, \alpha_{1} Z_{t}^{+}-\beta_{1} Z_{t}^{-} \leq \phi_{1}\left|Z_{t}\right|$ and, by Lyapunov's inequality, $E\left(\left|Z_{t}\right|\right) \leq \sqrt{E\left(Z_{t}^{2}\right)}=1$.

Therefore, $\frac{\alpha_{0}}{\sigma_{Y}}+\phi_{1} \geq 1 \Leftrightarrow \frac{1}{\phi_{1}}\left(1-\frac{\alpha_{0}}{\sigma_{Y}}\right) \leq 1$ and choosing $c \leq \frac{1}{\phi_{1}}\left(1-\frac{\alpha_{0}}{\sigma_{Y}}\right)$ such that $2 f_{|Z|}(x)+x f_{|Z|}^{\prime}(x) \geq 0$, where $x=\frac{c \sigma_{Y}}{\alpha_{0}+u}$, with $u \geq 0$, we have

$$
\begin{aligned}
& \frac{\alpha_{0}}{\sigma_{Y}}+c \phi_{1} \leq 1 \Leftrightarrow \frac{c}{\frac{\alpha_{0}}{\sigma_{Y}}+c \phi_{1}} \geq c \Leftrightarrow \\
\Leftrightarrow & \frac{1}{1-F_{|Z|}\left(\frac{c}{\frac{\alpha_{0}}{\sigma_{Y}}+c \phi_{1}}\right)} \geq \frac{1}{1-F_{|Z|}(c)} \Leftrightarrow \underset{\operatorname{TARCH}(1)}{L B_{1} A R L} \geq A R L_{i i d} .
\end{aligned}
$$

\section{Simulation study}

The goal of the simulation work, as mentioned, is to evaluate and compare the bounds obtained for the in-control $A R L$ of the modified Shewhart chart for $\mathrm{ARCH}$ and TARCH processes. We consider, in both cases, the first order models and present the results in two separate subsections. For $Z_{t}$ we take, in the case of the ARCH model, the standard normal distribution, $N(0,1)$, and, for the TARCH model, we consider the standard normal distribution and the unit variance distribution based on the Student's $t$-distribution with 6 degrees of freedom, $t(6)$. The study for the ARCH process follows Severin and Schmid (1999). 


\section{$5.1 \operatorname{ARCH}(1)$}

We consider the bounds below, already appropriately particularized for the case where the target process, $Y$, follows a stationary $\mathrm{ARCH}(1)$ model, that is $\alpha_{1}<1$ :

(i) $\underset{\operatorname{ARCH}(1)}{U B_{1} A R L}=\frac{1}{1-F_{Z^{2}}\left(\frac{c^{2}}{1-\alpha_{1}}\right)}$ valid for every $c \in \mathbb{R}^{+}$;

(ii) $\underset{\operatorname{ARCH}(1)}{L B_{1} A R L}=1+\frac{P\left(\left|Y_{1}\right| \leq c \sigma_{Y}\right)}{1-F_{Z^{2}}\left(\frac{c^{2}}{1+\left(c^{2}-1\right) \alpha_{1}}\right)}$, valid for every $c \in \mathbb{R}^{+}$;

(iii) $\underset{\mathrm{ARCH}(1)}{L B_{2} A R L}=\frac{1}{1-F_{Z^{2}}\left(c^{2}\right)}$, valid for every $c \in \mathbb{R}^{+}$such that $2 f_{Z^{2}}(x)+$ $x f_{Z^{2}}^{\prime}(x) \geq 0$, where $x=\frac{c^{2} \sigma_{Y}^{2}}{\alpha_{0}+u}$, with $u \geq 0 ;$

(iv) $\underset{\operatorname{ARCH}(1)}{L B_{3} A R L}=1+\frac{F_{Z^{2}}\left(c^{2}\right)}{1-F_{Z^{2}}\left(\frac{c^{2}}{1+\left(c^{2}-1\right) \alpha_{1}}\right)}$, valid for every $c \in \mathbb{R}^{+}$such that $2 f_{Z^{2}}(x)+x f_{Z^{2}}^{\prime}(x) \geq 0$, where $x=\frac{c^{2} \sigma_{Y}^{2}}{\alpha_{0}+u}$, with $u \geq 0$.

We considered $\alpha_{0}=1, \alpha_{1}=0,0.1,0.2,0.3,0.5,0.6,0.8$ and $Z_{t} \sim N(0,1)$. For each parameterization, we generated 25000 trajectories of 150 observations of the corresponding process $Y$. They were used to estimate the $A R L(\widehat{A R L})$, $P\left(\left|Y_{1}\right| \leq c \sigma_{Y}\right)$ and, consequently, $\left.\underset{\mathrm{ARCH}(1)}{\operatorname{Li} B_{1} A R L} \underset{\mathrm{ARCH}(1)}{\left(\underset{B_{1} A R}{A} L\right.}\right)$. The first 50 observations were discarded to eliminate the effect of choosing the value of the first observation.

In table 1 we report the results obtained in this simulation study, for $c=0.5$ and $c=0.8$, namely, the value of the $L B_{2} A R L, L B_{3} A R L$ and $U B_{1} A R L$ and the estimated $L B_{1} A R L$ and $A R L$. In each table, for the estimated values, in $\operatorname{ARCH}(1)$

the line immediately under, we give radius of the $95 \%$ confidence interval. The values typed in italic are the ones where the validity of the bounds cannot be ensured analytically. As seen on example 1 , we have ensured the validity of the lower bounds $L B_{2} A R L$ and $L B_{3} A R L$ when $0<c \leq \sqrt{3\left(1-\alpha_{1}\right)}$.

$$
\operatorname{ARCH}(1) \quad \operatorname{ARCH}(1)
$$

We observe that $\widehat{A R L}, L \widehat{B_{1} A R} L$ and $L B_{3} A R L$ increase with $\alpha_{1}$, but

$$
\operatorname{ARCH}(1) \quad \operatorname{ARCH}(1)
$$

$L B_{2} A R L$ maintains its value. For example, for $c=0.5$ and $0<\alpha_{1} \leq 0.5$, $\operatorname{ARCH}(1)$

the deviation of $L B_{3} A R L$ from $\widehat{A R L}$ is at most $7 \%$. 
Table 1 In-control $A R L$ of the modified Shewhart chart for an ARCH(1) process (with

\begin{tabular}{|c|c|c|c|c|c|c|c|c|}
\hline$c$ & $\alpha_{1}$ & 0.0 & 0.1 & 0.2 & 0.3 & 0.5 & 0.6 & 0.8 \\
\hline \multirow[t]{7}{*}{0.5} & $\begin{array}{c}L B_{2} A R L \\
\operatorname{ARCH}(1)\end{array}$ & 1.621 & 1.621 & 1.621 & 1.621 & 1.621 & 1.621 & 1.621 \\
\hline & $\begin{array}{c}L B_{3} A R L \\
\operatorname{ARCH}(1)\end{array}$ & 1.621 & 1.635 & 1.652 & 1.672 & 1.726 & 1.766 & 1.892 \\
\hline & $\begin{array}{c}L \widehat{B_{1} A R} L \\
\operatorname{ARCH}(1)\end{array}$ & 1.615 & 1.639 & 1.661 & 1.705 & 1.831 & 1.915 & 2.297 \\
\hline & CI $95 \%$ & \pm 0.010 & \pm 0.010 & \pm 0.010 & \pm 0.011 & \pm 0.012 & \pm 0.012 & \pm 0.014 \\
\hline & $\widehat{A R L}$ & 1.623 & 1.648 & 1.669 & 1.729 & 1.884 & 2.011 & 2.736 \\
\hline & CI $95 \%$ & \pm 0.013 & \pm 0.013 & \pm 0.013 & \pm 0.015 & \pm 0.017 & \pm 0.019 & \pm 0.031 \\
\hline & $\begin{array}{c}U B_{1} A R L \\
\operatorname{ARCH}(1)\end{array}$ & 1.621 & 1.672 & 1.736 & 1.818 & 2.086 & 2.330 & 3.794 \\
\hline \multirow[t]{7}{*}{0.8} & $\begin{array}{c}L B_{2} A R L \\
\operatorname{ARCH}(1)\end{array}$ & 2.360 & 2.360 & 2.360 & 2.360 & 2.360 & 2.360 & 2.360 \\
\hline & $\begin{array}{c}L B_{3} A R L \\
\operatorname{ARCH}(1)\end{array}$ & 2.360 & 2.388 & 2.418 & 2.452 & 2.529 & 2.573 & 2.680 \\
\hline & $\begin{array}{c}L \widehat{B_{1} A R L} L \\
\operatorname{ARCH}(1)\end{array}$ & 2.360 & 2.395 & 2.433 & 2.499 & 2.691 & 2.802 & 3.214 \\
\hline & CI $95 \%$ & \pm 0.014 & \pm 0.015 & \pm 0.015 & \pm 0.015 & \pm 0.016 & \pm 0.016 & \pm 0.015 \\
\hline & $\widehat{A R L}$ & 2.370 & 2.455 & 2.522 & 2.682 & 3.148 & 3.547 & 5.903 \\
\hline & CI $95 \%$ & \pm 0.022 & \pm 0.024 & \pm 0.025 & \pm 0.028 & \pm 0.034 & \pm 0.040 & \pm 0.073 \\
\hline & $\begin{array}{c}U B_{1} A R L \\
\operatorname{ARCH}(1)\end{array}$ & 2.360 & 2.506 & 2.695 & 2.950 & 3.877 & 4.857 & 13.580 \\
\hline
\end{tabular}

\section{$5.2 \operatorname{TARCH}(1)$}

We consider now the bounds indicated next, already properly particularized for the case where the target process $Y$ is a TARCH(1) process:

(i) $\underset{\operatorname{TARCH}(1)}{U B_{1} A R L}=\frac{1}{1-F_{|Z|}\left(\frac{c \sigma_{Y}}{\alpha_{0}}\right)}$, valid for every $c \in \mathbb{R}^{+}$;

(ii) $\underset{\operatorname{TARCH}(1)}{L B_{1} A R L}=1+\frac{F_{|Z|}(c)}{1-F_{|Z|}\left(\frac{c}{\frac{\alpha_{0}}{\sigma_{Y}}+c \max \left\{\alpha_{1}, \beta_{1}\right\}}\right)}$, valid for every $c \in \mathbb{R}^{+}$such that $2 f_{|Z|}(x)+x f_{|Z|}^{\prime}(x) \geq 0$, where $x=\frac{c \sigma_{Y}}{\alpha_{0}+u}$, with $u \geq 0$.

We note that the TARCH(1) model such that $Z_{t}$ has a symmetrical distribution, as are the cases considered, is stationary if $\alpha_{1}^{2}+\beta_{1}^{2}<2$.

For each parameterization indicated in tables 2 to 4 , we generated 25000 trajectories of 150 observations of the process $Y \sim \operatorname{TARCH}(1)$ such that $\alpha_{0}=1$. They were used to estimate the $A R L(\widehat{A R L})$. In each table, the $95 \%$ confidence interval of $\widehat{A R L}$ is given. As in the previous study, the first 50 observations were discarded.

Since the distributions considered for $Z_{t}$ are symmetrical, the $A R L$ and its bounds are symmetrical in $\alpha_{1}$ and $\beta_{1}$, meaning that, for example, the cases $\left(\alpha_{1}, \beta_{1}\right)=(0,0.1)$ and $\left(\alpha_{1}, \beta_{1}\right)=(0.1,0)$ produce the same results. So, we only present one of them, leaving the other one blank in the tables. 
Table 2 In-control $A R L$ of the modified Shewhart chart, with $c=0.5$, for a TARCH(1) process (with $\alpha_{0}=1$ and $Z_{t} \sim N(0,1)$ ).

\begin{tabular}{|c|c|c|c|c|c|c|c|}
\hline$\alpha_{1}$ & & 0 & 0.1 & 0.3 & 0.5 & 0.7 & 0.9 \\
\hline & $\begin{array}{l}L B_{1} A R L \\
\text { TARCH(1) }\end{array}$ & 1.621 & 1.618 & 1.615 & 1.616 & 1.620 & 1.628 \\
\hline \multirow[t]{4}{*}{0} & $\widehat{A R L}$ & 1.617 & 1.635 & 1.686 & 1.783 & 1.936 & 2.260 \\
\hline & CI $95 \%$ & \pm 0.013 & \pm 0.013 & \pm 0.014 & \pm 0.015 & \pm 0.017 & \pm 0.022 \\
\hline & $\begin{array}{l}U B_{1} A R L \\
\operatorname{TARCH}(1)\end{array}$ & 1.621 & 1.661 & 1.773 & 1.950 & 2.256 & 2.897 \\
\hline & $\begin{array}{l}L B_{1} A R L \\
\text { TARCH(1) }\end{array}$ & - & 1.632 & 1.628 & 1.628 & 1.631 & 1.638 \\
\hline \multirow[t]{4}{*}{0.1} & $\widehat{A R L}$ & - & 1.649 & 1.674 & 1.781 & 1.941 & 2.264 \\
\hline & CI $95 \%$ & ـ & \pm 0.013 & \pm 0.014 & \pm 0.015 & \pm 0.018 & \pm 0.023 \\
\hline & $\begin{array}{l}U B_{1} A R L \\
\operatorname{TARCH}(1)\end{array}$ & - & 1.706 & 1.829 & 2.025 & 2.372 & 3.118 \\
\hline & $\begin{array}{l}L B_{1} A R L \\
\text { TARCH(1) }\end{array}$ & - & - & 1.663 & 1.660 & 1.661 & 1.667 \\
\hline \multirow[t]{4}{*}{0.3} & $\widehat{A R L}$ & - & $\longrightarrow$ & 1.748 & 1.831 & 2.000 & 2.371 \\
\hline & CI $95 \%$ & 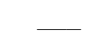 & $\ldots$ & \pm 0.015 & \pm 0.017 & \pm 0.019 & \pm 0.025 \\
\hline & $\begin{array}{l}U B_{1} A R L \\
\text { TARCH }(1)\end{array}$ & - & - & 1.986 & 2.248 & 2.737 & 3.904 \\
\hline & $\begin{array}{l}L B_{1} A R L \\
\text { TARCH(1) }\end{array}$ & 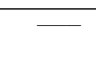 & - & - & - & 1.776 & 1.778 \\
\hline \multirow[t]{3}{*}{0.7} & $\widehat{A R L}$ & - & 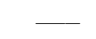 & - & - & 2.452 & 3.217 \\
\hline & CI $95 \%$ & - & - & - & 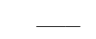 & \pm 0.027 & \pm 0.038 \\
\hline & $\begin{array}{l}U B_{1} A R L \\
\operatorname{TARCH}(1)\end{array}$ & - & $\longrightarrow$ & $\longrightarrow$ & - & 5.309 & 13.877 \\
\hline
\end{tabular}

In table 2 we report the results when $Z_{t} \sim N(0,1)$. As detailed in example 3 (a), we need to have $0<c \leq \frac{\sqrt{2} \alpha_{0}}{\sigma_{Y}}$ so that we can ensure analytically the validity of $L B_{1} A R L$. In tables 3 and 4 we report the results when $\sqrt{\frac{3}{2}} Z_{t} \sim t(6)$. As described in example 3 (b), we have guaranteed analytically the validity of $L B_{1} A R L$ if $0<c \leq \frac{\sqrt{1.6} \alpha_{0}}{\sigma_{Y}}$. When $c$ exceeds the indicated value, the $L B_{1} A R L$ TARCH(1) is typed in italic. Nevertheless, we observe, in tables 2 to 4 , that all of these values are still smaller than the $\widehat{A R L}$. This suggests that there might be less restrictive conditions than those associated to $2 f_{|Z|}(x)+x f_{|Z|}^{\prime}(x) \geq 0$.

Moreover, in tables 2 to 4 , if we fix $\alpha_{1}$, as $\beta_{1}$ increases the $\widehat{A R L}$ and $U B_{1} A R L$ increase too. But the $L B_{1} A R L$ does not perform as well, since, for TARCH(1) TARCH(1) $c=0.5$, if we fix $\alpha_{1}$, as $\beta_{1}$ increases its value is virtually unchanged and, for $c=1$, decreases. However, we can state that the accuracy of the bounds improves as $c, \alpha_{1}$ and $\beta_{1}$ are closer to zero, regardless of the distribution considered. 
Table 3 In-control $A R L$ of the modified Shewhart chart, with $c=0.5$, for a TARCH(1) process (with $\alpha_{0}=1$ and $\sqrt{\frac{3}{2}} Z_{t} \sim t(6)$ ).

\begin{tabular}{|c|c|c|c|c|c|c|c|}
\hline$\alpha_{1}$ & & 0 & 0.1 & 0.3 & 0.5 & 0.7 & 0.9 \\
\hline \multirow{4}{*}{0} & $\begin{array}{l}L B_{1} A R L \\
\text { TARCH(1) }\end{array}$ & 1.777 & 1.771 & 1.765 & 1.765 & 1.770 & 1.781 \\
\hline & $\widehat{A R L}$ & 1.780 & 1.789 & 1.846 & 1.972 & 2.170 & 2.546 \\
\hline & CI $95 \%$ & \pm 0.015 & \pm 0.015 & \pm 0.016 & \pm 0.018 & \pm 0.021 & \pm 0.026 \\
\hline & $\begin{array}{l}U B_{1} A R L \\
\operatorname{TARCH}(1)\end{array}$ & 1.777 & 1.827 & 1.964 & 2.179 & 2.551 & 3.307 \\
\hline \multirow{4}{*}{0.3} & $\begin{array}{l}L B_{1} A R L \\
\text { TARCH }(1)\end{array}$ & - & - & 1.832 & 1.825 & 1.826 & 1.833 \\
\hline & $\widehat{A R L}$ & - & - & 1.941 & 2.051 & 2.288 & 2.713 \\
\hline & CI $95 \%$ & - & 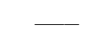 & \pm 0.018 & \pm 0.019 & \pm 0.023 & \pm 0.030 \\
\hline & $\begin{array}{l}U B_{1} A R L \\
\operatorname{TARCH}(1)\end{array}$ & 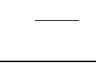 & - & 2.220 & 2.532 & 3.101 & 4.376 \\
\hline \multirow{4}{*}{0.7} & $\begin{array}{l}L B_{1} A R L \\
\text { TARCH }(1)\end{array}$ & 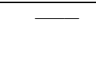 & $\bar{\square}$ & $\overline{-}$ & - & 1.983 & 1.987 \\
\hline & $\widehat{A R L}$ & 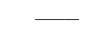 & $\longrightarrow$ & - & $\longrightarrow$ & 2.826 & 3.796 \\
\hline & CI $95 \%$ & - & 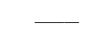 & - & - & \pm 0.032 & \pm 0.046 \\
\hline & $\begin{array}{l}U B_{1} A R L \\
\operatorname{TARCH}(1)\end{array}$ & 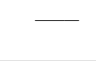 & 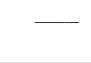 & 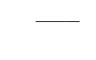 & - & 5.703 & 11.924 \\
\hline
\end{tabular}

Table 4 In-control $A R L$ of the modified Shewhart chart, with $c=1$, for a TARCH(1) process (with $\alpha_{0}=1$ and $\sqrt{\frac{3}{2}} Z_{t} \sim t(6)$ ).

\begin{tabular}{|c|c|c|c|c|c|c|}
\hline$\alpha_{1}$ & & 0 & 0.1 & 0.3 & 0.5 & 0.7 \\
\hline & $\begin{array}{l}L B_{1} A R L \\
\text { TARCH }(1)\end{array}$ & 3.751 & 3.510 & 3.179 & 2.964 & 2.816 \\
\hline \multirow[t]{4}{*}{0} & $\widehat{A R L}$ & 3.731 & 3.851 & 4.107 & 4.621 & 5.477 \\
\hline & CI $95 \%$ & \pm 0.039 & \pm 0.041 & \pm 0.045 & \pm 0.051 & \pm 0.063 \\
\hline & $\begin{array}{l}U B_{1} A R L \\
\text { TARCH(1) }\end{array}$ & 3.751 & 4.007 & 4.759 & 6.078 & 8.718 \\
\hline & $\begin{array}{l}L B_{1} A R L \\
\text { TARCH }(1)\end{array}$ & - & - & 3.517 & 3.190 & 2.975 \\
\hline \multirow[t]{4}{*}{0.3} & $\widehat{A R L}$ & - & - & 4.479 & 4.957 & 5.946 \\
\hline & CI $95 \%$ & 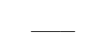 & 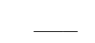 & \pm 0.051 & \pm 0.057 & \pm 0.070 \\
\hline & $\begin{array}{l}U B_{1} A R L \\
\text { TARCH }(1)\end{array}$ & - & - & 6.344 & 8.572 & 13.423 \\
\hline & $\begin{array}{l}L B_{1} A R L \\
\text { TARCH(1) }\end{array}$ & - & $\overline{-}$ & - & - & 3.385 \\
\hline \multirow[t]{3}{*}{0.7} & $\widehat{A R L}$ & - & - & - & - & 8.102 \\
\hline & CI $95 \%$ & - & - & - & - & \pm 0.101 \\
\hline & $\begin{array}{l}U B_{1} A R L \\
\text { TARCH }(1)\end{array}$ & - & - & - & - & 45.744 \\
\hline
\end{tabular}




\section{Appendix. Proof of Theorem 1}

(b) Let us begin by the case $n>q$. We start by observing that, if $Y_{1}^{2} \leq$ $x_{1}, \ldots, Y_{n}^{2} \leq x_{n}$, then:

(i) for $t \in\{2, \ldots, q\}$,

$$
\sigma_{t}^{2}=\alpha_{0}+\sum_{i=1}^{t-1} \alpha_{i} Y_{t-i}^{2}+\sum_{i=t}^{q} \alpha_{i} Y_{t-i}^{2} \leq \alpha_{0}+\sum_{i=1}^{t-1} \alpha_{i} x_{t-i}+\sum_{i=t}^{q} \alpha_{i} Y_{t-i}^{2}
$$

since, for $i \in\{1, \ldots, t-1\}, 1 \leq t-i \leq q-1<n$ and, for $i \in\{t, \ldots, q\}$, $t-i \leq 0$

(ii) and, for $t \in\{q+1, \ldots, n\}$,

$$
\sigma_{t}^{2}=\alpha_{0}+\sum_{i=1}^{q} \alpha_{i} Y_{t-i}^{2} \leq \alpha_{0}+\sum_{i=1}^{q} \alpha_{i} x_{t-i}
$$

as, for $i \in\{1, \ldots, q\}, 1 \leq t-i \leq n-1$.

So, for every $\left.\left(x_{1}, \ldots, x_{n}\right) \in\right] 0,+\infty\left[^{n}\right.$,

$$
\begin{aligned}
& P\left(Y_{t}^{2} \leq x_{t}, t=1, \ldots, n\right) \geq \\
& \geq P\left(Z_{1}^{2} \leq \frac{x_{1}}{\sigma_{1}^{2}}, Z_{t}^{2} \leq \frac{x_{t}}{\alpha_{0}+\sum_{i=1}^{t-1} \alpha_{i} x_{t-i}+\sum_{i=t}^{q} \alpha_{i} Y_{t-i}^{2}}, t=2, \ldots, q,\right. \\
& =E\left[P \left(Z_{1}^{2} \leq \frac{x_{1}}{\sigma_{1}^{2}}, Z_{t}^{2} \leq \frac{x_{t}+\sum_{i=1}^{q} \alpha_{i} x_{t-i}}{\alpha_{0}+\sum_{i=1}^{t-1} \alpha_{i} x_{t-i}+\sum_{i=t}^{q} \alpha_{i} Y_{t-i}^{2}}, t=q, \ldots, q,\right.\right. \\
& \left.\left.Z_{t}^{2} \leq \frac{x_{t}}{\alpha_{0}+\sum_{i=1}^{q} \alpha_{i} x_{t-i}}, t=q+1, \ldots, n \mid \underline{Y}_{0}\right)\right]
\end{aligned}
$$

As $Z_{t}$ is independent of $\underline{Y}_{t-1}$ and $\underline{Y}_{0} \subseteq \underline{Y}_{t-1}(t=1,2, \ldots)$ and $Z_{1}, \ldots, Z_{n}$ are iid, it holds, according to the expectation and conditional expectation 
properties,

$$
\begin{aligned}
& P\left(Y_{t}^{2} \leq x_{t}, t=1, \ldots, n\right) \geq \\
& \geq E\left[F_{Z^{2}}\left(\frac{x_{1}}{\sigma_{1}^{2}}\right) \prod_{t=2}^{q} F_{Z^{2}}\left(\frac{x_{t}}{\alpha_{0}+\sum_{i=1}^{t-1} \alpha_{i} x_{t-i}+\sum_{i=t}^{q} \alpha_{i} Y_{t-i}^{2}}\right) F_{q, n}\right] \\
& \quad=E\left[F_{Z^{2}}\left(\frac{x_{1}}{\sigma_{1}^{2}}\right)\right] \prod_{t=2}^{q} E\left[F_{Z^{2}}\left(\frac{x_{t}}{\alpha_{0}+\sum_{i=1}^{t-1} \alpha_{i} x_{t-i}+\sum_{i=t}^{q} \alpha_{i} Y_{t-i}^{2}}\right)\right] F_{q, n},
\end{aligned}
$$

where

$$
F_{q, n}=\prod_{t=q+1}^{n} F_{Z^{2}}\left(\frac{x_{t}}{\alpha_{0}+\sum_{i=1}^{q} \alpha_{i} x_{t-i}}\right) \text {. }
$$

For $t$ arbitrarily fixed in $\{1, \ldots, q\}$, we consider the function $R_{t}:[0,+\infty[\longrightarrow[0,1]$ defined by

$$
R_{t}\left(u_{t}\right)=F_{Z^{2}}\left(\frac{x_{t}}{\alpha_{0}+\sum_{i=1}^{t-1} \alpha_{i} x_{t-i}+u_{t}}\right)
$$

Let $m_{t}=\alpha_{0}+\sum_{i=1}^{t-1} \alpha_{i} x_{t-i}+u_{t}$. We have

$$
\frac{d^{2} R_{t}}{d u_{t}^{2}}\left(u_{t}, \ldots, u_{q}\right)=\frac{x_{t}}{m_{t}^{3}}\left[2 f_{Z^{2}}\left(\frac{x_{t}}{m_{t}}\right)+\frac{x_{t}}{m_{t}} f_{Z^{2}}^{\prime}\left(\frac{x_{t}}{m_{t}}\right)\right] ;
$$

hence, if $2 f_{Z^{2}}\left(\frac{x_{t}}{m_{t}}\right)+\frac{x_{t}}{m_{t}} f_{Z^{2}}^{\prime}\left(\frac{x_{t}}{m_{t}}\right) \geq 0$, where $m_{t}=\alpha_{0}+u$, with $u \geq 0, R_{t}$ is a convex function. Therefore, if, for each $x_{t}, t \in\{1, \ldots, q\}, \frac{x_{t}}{\alpha_{0}+u} f_{Z^{2}}^{\prime}\left(\frac{x_{t}}{\alpha_{0}+u}\right)+$ $2 f_{Z^{2}}\left(\frac{x_{t}}{\alpha_{0}+u}\right) \geq 0$, with $u \geq 0$, we can apply Jensen's inequality and obtain

$$
\begin{aligned}
& P\left(Y_{t}^{2} \leq x_{t}, t=1, \ldots, n\right) \geq \\
& \quad \geq F_{Z^{2}}\left(\frac{x_{1}}{E\left(\sigma_{1}^{2}\right)}\right) \prod_{t=2}^{q} F_{Z^{2}}\left(\frac{x_{t}}{\alpha_{0}+\sum_{i=1}^{t-1} \alpha_{i} x_{t-i}+\sum_{i=t}^{q} \alpha_{i} E\left(Y_{t-i}^{2}\right)}\right) F_{q, n} .
\end{aligned}
$$

So, as, for all $t \in \mathbb{Z}, E\left(Y_{t}^{2}\right)=E\left(\sigma_{1}^{2}\right)=V\left(Y_{t}\right)=\sigma_{Y}^{2}$, we conclude that

$$
P\left(Y_{t}^{2} \leq x_{t}, t=1, \ldots, n\right) \geq F_{Z^{2}}\left(\frac{x_{1}}{\sigma_{Y}^{2}}\right) \prod_{t=2}^{q} F_{Z^{2}}\left(\frac{x_{t}}{\alpha_{0}+\sum_{i=1}^{t-1} \alpha_{i} x_{t-i}+\sigma_{Y}^{2} \sum_{i=t}^{q} \alpha_{i}}\right) F_{q, n} .
$$


(a) Let us now turn to the case $n \leq q$. In this case, if $Y_{1}^{2} \leq x_{1}, \ldots, Y_{n}^{2} \leq x_{n}$, then, for $t \in\{2, \ldots, n\}$,

$$
\sigma_{t}^{2}=\alpha_{0}+\sum_{i=1}^{t-1} \alpha_{i} Y_{t-i}^{2}+\sum_{i=t}^{q} \alpha_{i} Y_{t-i}^{2} \leq \alpha_{0}+\sum_{i=1}^{t-1} \alpha_{i} x_{t-i}+\sum_{i=t}^{q} \alpha_{i} Y_{t-i}^{2}
$$

as, for $i \in\{1, \ldots, t-1\}, 1 \leq t-i \leq n-1$ and, for $i \in\{t, \ldots, q\}, t-i \leq 0$.

Hence, for every $\left.\left(x_{1}, \ldots, x_{n}\right) \in\right] 0,+\infty\left[^{n}\right.$,

$$
\begin{aligned}
& P\left(Y_{t}^{2} \leq x_{t}, t=1, \ldots, n\right) \geq \\
& \quad \geq P\left(Z_{1}^{2} \leq \frac{x_{1}}{\sigma_{1}^{2}}, Z_{t}^{2} \leq \frac{x_{t}}{\alpha_{0}+\sum_{i=1}^{t-1} \alpha_{i} x_{t-i}+\sum_{i=t}^{q} \alpha_{i} Y_{t-i}^{2}}, t=2, \ldots, n\right) .
\end{aligned}
$$

Following the same line of reasoning of the previous case, we can state that, if, for every $x_{t}, t \in\{1, \ldots, n\}, \frac{x_{t}}{\alpha_{0}+u} f_{Z^{2}}^{\prime}\left(\frac{x_{t}}{\alpha_{0}+u}\right)+2 f_{Z^{2}}\left(\frac{x_{t}}{\alpha_{0}+u}\right) \geq 0$, with $u \geq 0$, then

$P\left(Y_{t}^{2} \leq x_{t}, t=1, \ldots, n\right) \geq F_{Z^{2}}\left(\frac{x_{1}}{\sigma_{Y}^{2}}\right) \prod_{t=2}^{n} F_{Z^{2}}\left(\frac{x_{t}}{\alpha_{0}+\sum_{i=1}^{t-1} \alpha_{i} x_{t-i}+\sigma_{Y}^{2} \sum_{i=t}^{q} \alpha_{i}}\right)$.

Acknowledgements We thank the referee for the constructive suggestions.

\section{References}

[1] Gonçalves E, Mendes-Lopes N (1994) The generalized threshold ARCH model: wide sense stationarity and asymptotic normality of the temporal aggregate. Pub Inst Stat Univ Paris XXXVIII:19-35

[2] Gonçalves E, Mendes-Lopes N (2007) On the distribution of generalized threshold ARCH stochastic processes. Int J Pure Appl Math 35:397-419

[3] Engle RF (1982) Autoregressive conditional heteroscedasticity with estimates of the variance of United Kingdom inflation. Econometrica 50:987-1007

[4] Pawlak M, Schmid W (2001) On the distributional properties of GARCH processes. J Time Ser Anal 22:339-352

[5] Schmid W (1995) On the run length of a Shewhart chart for correlated data. Stat Papers 36:111-130

[6] Severin T, Schmid W (1999) Monitoring changes in GARCH processes. Allg Stat Arch 83:281-307

[7] Shewhart W (1931) Economic control of quality of manufactured product. Van Nostrand, New York

[8] Vasilopoulos AV, Stamboulis AP (1978) Modification of control chart limits in the presence of data correlation. J Qual Technol 10:20-30

[9] Zakoian JM (1994) Threshold heteroskedasticity models. J Econ Dyn Control 18:931-955 